\title{
Investigation of Velocity Distribution in Open Channel Flows Based on Conditional Average of Turbulent Structures
}

\author{
Yu Han, ${ }^{1}$ Shu-Qing Yang, ${ }^{2}$ Muttucumaru Sivakumar, ${ }^{2}$ and Liu-Chao Qiu ${ }^{1}$ \\ ${ }^{1}$ College of Water Resources \& Civil Engineering, China Agricultural University, Beijing 100083, China \\ ${ }^{2}$ School of Civil, Mining \& Environmental Engineering, University of Wollongong, NSW 2522, Australia \\ Correspondence should be addressed to Yu Han; yh916@uowmail.edu.au and Liu-Chao Qiu; qiuliuchao@cau.edu.cn
}

Received 4 January 2017; Accepted 24 April 2017; Published 29 May 2017

Academic Editor: Jian G. Zhou

Copyright (c) 2017 Yu Han et al. This is an open access article distributed under the Creative Commons Attribution License, which permits unrestricted use, distribution, and reproduction in any medium, provided the original work is properly cited.

\begin{abstract}
We report the development of a new analytical model similar to the Reynolds-averaged Navier-Stokes equations to determine the distribution of streamwise velocity by considering the bursting phenomenon. It is found that, in two-dimensional (2D) flows, the underlying mechanism of the wake law in $2 \mathrm{D}$ uniform flow is actually a result of up/down events. A special experiment was conducted to examine the newly derived analytical model, and good agreement is achieved between the experimental data in the inner region and the model's prediction. The obtained experimental data were also used to examine the DML-Law (dip-modifiedlog-law), MLW-Law (modified-log-wake law), and CML-Law (Cole's wake law), and the agreement is not very satisfactory in the outer region.
\end{abstract}

\section{Introduction}

Velocity distribution in open channel flows offers a wide range of applications in the fields of hydrometry, sediment transport, river restoration, power plant design, and so forth [1-3]. Accurate investigation of the velocity distribution in open channel flows has been conducted over the past century. The law of the wall, developed for pipe flows, has often been applied to uniform flows in open channel flows [4]; this "universal wall function" or log-law of the wall is expressed as

$$
\frac{u}{u_{*}}=\frac{1}{\kappa} \ln \frac{y}{y_{0}}
$$

and herein $\kappa$ is the von Karman constant, $u_{*}$ is the mean friction velocity, and $y$ is the vertical direction. In (1), the boundary condition is $u=0$ at $y=y_{0}$ [5], and the parameter $y_{0}$ is the constant of integration that must be determined using the boundary condition [6]. It is expected that the parameter $y_{0}$ in (1) should be a variable as well [7], for a smooth boundary $y_{0}$ should be related to the thickness of the local viscous sublayer; that is,

$$
y_{0}=\frac{v}{c u_{* \mathbf{s}}}
$$

where $c$ is the coefficient to be determined experimentally, $v$ is the kinematic viscosity, and $u_{* s}$ denotes the local friction velocity, which may be different from $u_{*}$.

The velocity vertical profile is well described by the classical log-law in the inner region, $y / h<0.2$, but the loglaw normally deviates from the experimental data in the outer ration, $y / h>0.2$ [8]. Since this method is unable to represent the outer region of a velocity profile, Coles [9] suggested an improvement, called the wake law. Many researchers have discussed the validity of such a law $[10,11]$ and have improved both the log and wake laws for smooth or rough flows [12,13].

Coles [9] extended the log-law by introducing a purely empirical correction function. He developed an additional term (i.e., the wake term) as a supplement to the log-law, to account for the velocity deviation caused by wake. This model is known as the wake law:

$$
\frac{u}{u_{*}}=\frac{1}{\kappa} \ln \frac{y}{y_{0}}+\frac{2 \Pi}{\kappa} \sin ^{2}\left(\frac{\pi y}{2 h}\right),
$$

where $\Pi$ denotes the "profile" parameter; Coles [9] gave $\Pi$ $=0.55$. For open channel flows, Coleman [14] obtained an average $\Pi$ value of 0.19 . Nezu and Rodi [15] yielded a $\Pi$ value of 0 to 0.20 , while Kirkgöz [16] reported a value of 
0.1. Cardoso et al. [10] obtained a $\Pi$ value of -0.077 over a smooth bed. Kironoto and Graf [11] found $\Pi$ value in the range of -0.08 to 0.15 for water flows over a gravel bed. However, the values of $\Pi$ are established by experimentation, with large variations reported in the literatures. While significant advances have been made by using Cole's wake law, the mechanism of Cole's wake law (CWL-Law) and the associated wake strength parameter are not fully understood. These investigations often ascribe the large variations to either smooth or rough boundaries and few researchers have taken into account the velocity-dip phenomenon caused by secondary currents [17] (Guo 2015).

Different from the positive deviation of measured velocity from the log-law's prediction or the CWL-Law, Yang et al. [18] discussed the negative deviation of measured velocity from the log-law's prediction or the dip phenomenon, in which the maximum longitudinal velocity occurs below the water surface. They suggested that Cole's wake law is not able to describe the entire velocity profile when the dip phenomenon exists. Yang et al. [18] proposed a dip-modified-log-law (DML-Law) based on the analysis of the Reynolds-averaged Navier-Stokes (RANS) equations. This law, involving two logarithmic distances, one from the bed (i.e., the log-law) and the other from the free surface, has the advantage that it contains only one parameter $\alpha$ for dip-correction. The DMLLaw reverts to the classical log-law for $\alpha=0$. Yang et al. [18] modified the log-law by adding a term to express the dip phenomenon instead of Cole's wake law based on Reynolds equations:

$$
\begin{aligned}
\frac{u}{u_{*}} & =\frac{1}{\kappa} \ln \frac{y}{y_{0}}+\frac{\alpha}{\kappa} \ln \left(1-\frac{y}{h}\right), \\
\alpha & =1.3 \exp \left(-\frac{b}{2 h}\right) .
\end{aligned}
$$

Guo and Julien [12] proposed a modified-log-wake law $(M L W-L a w)$ which fits velocity profiles with a dip phenomenon. However, this law cannot be used for predictive applications since it requires fitting the near-free-surface velocities to the parabolic law to obtain dip position and maximum velocity. As Guo and Julien indicate, the MLWLaw can be used only in flow measurements since it requires measured velocities; that is,

$$
\frac{u}{u_{*}}=\frac{1}{\kappa} \ln \frac{y}{y_{0}}+\frac{2 \Pi}{\kappa} \sin ^{2} \frac{\pi \varsigma}{2}-\frac{\varsigma^{3}}{3 \kappa},
$$

where $\varsigma=y / \delta$ and $\delta$ is the distance from the bed to the point where the velocity is maximum.

Nezu and Nakagawa [19] found that in the outer region the mean velocity data deviate systematically from the loglaw distribution; at sufficiently high Reynolds numbers, this deviation cannot be neglected in the free-surface region; that is, $y / h>0.6$. It is thus worth investigating the underlying mechanism of velocity deviation from the log-law. Bonakdari et al. [20] also proposed a new formulation of the vertical velocity profile in open channel flows based on an analysis of the Navier-Stoke equations. On the basis of this law and of the suggestions of Absi [21], a new analytical procedure was presented by Pu et al. [22].
With the advent of nonintrusive instrumentation, visualisation techniques reveal that turbulence is dominated by forceful structures that are well organised and ordered eddies with a certain lifespan, now termed as coherent structures. Coherent structures can be placed into two categories: bursting phenomena that occur in the wall region and large-scale vertical motion in the outer region. For coherent structural analysis, the method of quadrants has been developed based on measured velocity fluctuations, that is, quadrant I, $u^{\prime}>0$ and $v^{\prime}>0$; quadrant II, $u^{\prime}<0$ and $v^{\prime}>0$; quadrant III, $u^{\prime}<0$ and $v^{\prime}<0$; and quadrant IV, $u^{\prime}>0$ and $v^{\prime}<0$ [23]. Cellino and Lemmin [24] pointed out that it would be meaningful to concentrate on $v^{\prime}>0$ and $v^{\prime}<0$ in quadrants because the most significant feature of turbulence is fluctuations in the wall-normal direction. They also developed an approach to calculate conditionally averaged upward/downward velocity based on velocity fluctuations. However, the linkage of quadrant analysis and upward/downward events with velocity deviation from the log-law has not been established.

The objective of this research is to present a new analytical approach to determine the streamwise velocity profile and establish a relationship between the log wake function and up/down events. This leads to the present research aim to develop a universal model to express the velocity profile in uniform flows. Such a novel analytical approach will be used to explain that streamwise velocity profiles in the outer region depart from the universal logarithmic law of the wall. The proposed analytical approach and those previously proposed mathematical methods will be examined by using experimental data.

\section{Theoretical Consideration}

The RANS momentum and continuity equations can be written for steady state flow as

$$
\begin{aligned}
& u\left(\frac{\partial u}{\partial x}\right)+v\left(\frac{\partial u}{\partial y}\right)+w\left(\frac{\partial u}{\partial z}\right) \\
& =g S+\frac{\partial}{\partial x}\left(\overline{-u^{\prime} u^{\prime}}\right)+\frac{\partial}{\partial y}\left(\overline{-u^{\prime} v^{\prime}}\right)+\frac{\partial}{\partial z}\left(\overline{-u^{\prime} w^{\prime}}\right) \\
& \quad+v\left(\frac{\partial^{2} u}{\partial x^{2}}+\frac{\partial^{2} u}{\partial y^{2}}+\frac{\partial^{2} u}{\partial z^{2}}\right) \\
& \frac{\partial u}{\partial x}+\frac{\partial v}{\partial y}+\frac{\partial w}{\partial z}=0
\end{aligned}
$$

where $u, v$, and $w$ denote the mean velocity in the streamwise $(x)$, lateral $(y)$, and vertical $(z)$ directions, respectively, $g$ is the gravitational acceleration, $S$ is the energy slope, and $\overline{-u^{\prime} w^{\prime}}$ are the Reynolds stress tensor components. For a uniform and fully developed flow, (7) becomes

$$
\begin{aligned}
v\left(\frac{\partial u}{\partial y}\right)+w\left(\frac{\partial u}{\partial z}\right)= & g S+\frac{\partial}{\partial y}\left(\overline{-u^{\prime} v^{\prime}}\right)+\frac{\partial}{\partial z}\left(\overline{-u^{\prime} w^{\prime}}\right) \\
& +v\left(\frac{\partial^{2} u}{\partial y^{2}}+\frac{\partial^{2} u}{\partial z^{2}}\right) .
\end{aligned}
$$


Alternatively, (9) becomes

$$
\begin{aligned}
\frac{\partial\left(u v-\tau_{x y} / \rho\right)}{\partial y}+\frac{\partial\left(u v-\tau_{x z} / \rho\right)}{\partial z} & =g S \\
\tau_{x y} & =\mu \frac{\partial u}{\partial y}-\rho \overline{u^{\prime} v^{\prime}}, \\
\tau_{x z} & =\mu \frac{\partial u}{\partial z}-\rho \overline{u^{\prime} w^{\prime}},
\end{aligned}
$$

where $\rho$ is the density of the fluid, $\mu$ is the dynamic viscosity of the fluid, and $u^{\prime}, v^{\prime}$, and $w^{\prime}$ are the turbulent velocity fluctuation in the $x, y$, and $z$ directions. For 2D flow, (10) can be simplified as

$$
\frac{\partial\left(u v+\overline{u^{\prime} v^{\prime}}-v d u / d y\right)}{\partial y}=g S .
$$

Integration of (13) yields

$$
v \frac{d u}{d y}-u v-\overline{u^{\prime} v^{\prime}}=-g S y+C
$$

where $C$ is the integration constant. Using the boundary condition, $y=0, u=v=0, \overline{u^{\prime} v^{\prime}}=0$, and $v d u / d y=u_{*}^{2}$, and thus $C=u_{*}^{2}$. For water surface, $y=h, v=0$, and $\overline{u^{\prime} v^{\prime}}=0$ and then the shear velocity can be rewritten as

$$
u_{*}^{2}=g S h
$$

Therefore, the momentum equation has the following form by dividing (15):

$$
\frac{d u^{+}}{d y^{+}}-u^{+} v^{+}-\frac{\overline{u^{\prime} v^{\prime}}}{u_{*}^{2}}=1-\frac{y}{h},
$$

where $u^{+}=u / u_{*}$ and $v^{+}=v / u_{*}$.

Cellino and Lemmin [24] realised that it would be meaningful to develop an approach to calculate the distribution of streamwise velocity by considering the bursting phenomenon. However, the above time averaged method cannot be used to express the bursting phenomenon. Yang [25] proposed an approach to calculate the conditionally averaged upward/downward velocity based on velocity fluctuations. He first defines the occurrence probabilities $r$ based on the direction of vertical velocity. For upflow (subscript $u$ ) and downflow (subscript $d$ ), $r$ is expressed as

$$
\begin{aligned}
& r_{u}=\frac{\sum_{0}^{T_{u}} \Delta t_{u}}{T}, \\
& r_{d}=\frac{\sum_{0}^{T_{d}} \Delta t_{d}}{T},
\end{aligned}
$$

where $\Delta t$ is the time interval between two consecutive velocity acquisitions in an experiment; $T$ is the total observed time period; that is, $\sum \Delta t_{u}+\sum \Delta t_{d}=T_{u}+T_{d}=T$. The upflow and downflow velocity definitions are given in the following way:

$$
\begin{aligned}
& u_{u}=\frac{1}{T_{u}} \int_{0}^{T_{u}} \tilde{u}_{u} d t, \\
& u_{d}=\frac{1}{T_{d}} \int_{0}^{T_{d}} \tilde{u}_{d} d t, \\
& v_{u}=\frac{1}{T_{u}} \int_{0}^{T_{u}} \tilde{v}_{u} d t, \\
& v_{d}=\frac{1}{T_{d}} \int_{0}^{T_{d}} \tilde{v}_{d} d t,
\end{aligned}
$$

where $u_{u}$ and $u_{d}$ denote upflow and downflow events, respectively, and the symbol “ $\sim$ " represents instantaneous velocities. Yang [25] obtained the Reynolds shear stress for upflow and downflow as follows, and both deviate from the standard linear distribution; that is,

$$
\begin{array}{r}
-\frac{\overline{u_{u}^{\prime} v_{u}^{\prime}}}{u_{* u}^{2}}=\left(1-\frac{y}{h}\right)+u_{u}^{+} v_{u}^{+}, \\
-\frac{\overline{u_{d}^{\prime} v_{d}^{\prime}}}{u_{* d}^{2}}=\left(1-\frac{y}{h}\right)+u_{d}^{+} v_{d}^{+},
\end{array}
$$

where $u_{u}^{\prime}=\widetilde{u}-u_{u}, u_{d}^{\prime}=\widetilde{u}-u_{d}, u_{u}^{+}=u_{u} / u_{* u}$, and $u_{d}^{+}=u_{d} /$ $u_{* d} ; h$ is water depth; and $u_{* u}$ and $u_{* d}$ are friction velocities for upflow (subscript $u$ ) and downflow (subscript $d$ ), respectively.

The expression of eddy viscosity $v_{t}$ is

$$
\frac{v_{t}}{u_{*} h}=\kappa \xi(1-\xi) \text {, }
$$

where $\xi=y / h$ and (24) can be expressed as

$$
\kappa \xi(1-\xi) \frac{d u_{u}^{+}}{d \xi}=(1-\xi)+u_{u}^{+} v_{u}^{+} .
$$

The velocity gradient in upflow and downflow can then be expressed by

$$
\begin{aligned}
& \frac{d u_{u}^{+}}{d \xi}=\frac{1}{\kappa \xi}+\frac{u_{u}^{+} v_{u}^{+}}{\kappa \xi(1-\xi)}, \\
& \frac{d u_{d}^{+}}{d \xi}=\frac{1}{\kappa \xi}+\frac{u_{d}^{+} v_{d}^{+}}{\kappa \xi(1-\xi)} .
\end{aligned}
$$

Similarly, the gradient of mean velocity can be expressed by

$$
\frac{d u^{+}}{d \xi}=r_{u} \frac{u_{* u}}{u_{*}} \frac{d u_{u}^{+}}{d \xi}+r_{d} \frac{u_{* d}}{u_{*}} \frac{d u_{d}^{+}}{d \xi} .
$$

Inserting (26) into (27) yields

$$
\begin{aligned}
\frac{d u^{+}}{d \xi}= & \frac{1}{\kappa \xi}\left(r_{u} \frac{u_{* u}}{u_{*}}+r_{d} \frac{u_{* d}}{u_{*}}\right) \\
& +r_{u} \frac{v_{u}^{+}}{\kappa \xi(1-\xi)} \frac{u_{* u}}{u_{*}}\left(1-\frac{u_{d}^{+}}{u_{u}^{+}}\right) u_{u}^{+},
\end{aligned}
$$


TABLE 1: Summary of the main hydraulic parameters in rectangular channel.

\begin{tabular}{llccccccc}
\hline & Slope & Water depth, $h(\mathrm{~m})$ & Width, $b(\mathrm{~m})$ & $R(\mathrm{~m})$ & Aspect ratio, $b / h$ & $Q\left(\mathrm{~m}^{3} / \mathrm{s}\right)$ & $u_{*}=\sqrt{g R S}(\mathrm{~m} / \mathrm{s})$ & $u_{*}=\sqrt{g h S}(\mathrm{~m} / \mathrm{s})$ \\
\hline Run 1 & 0.001 & 0.065 & 0.300 & 0.045 & 4.615 & 0.008 & 0.021 & 0.025 \\
Run 2 & 0.001 & 0.085 & 0.300 & 0.054 & 3.529 & 0.012 & 0.023 & 0.029 \\
Run 3 & 0.001 & 0.100 & 0.300 & 0.060 & 3.000 & 0.015 & 0.024 & 0.031 \\
Run 4 & 0.001 & 0.110 & 0.300 & 0.063 & 2.727 & 0.017 & 0.025 & 0.033 \\
Run 5 & 0.001 & 0.150 & 0.300 & 0.075 & 2.000 & 0.025 & 0.027 & 0.038 \\
Run 6 & 0.001 & 0.160 & 0.300 & 0.077 & 1.875 & 0.028 & 0.028 & 0.040 \\
\hline
\end{tabular}

where $r_{u}\left(u_{* u} / u_{*}\right)+r_{d}\left(u_{* d} / u_{*}\right)=1$, and then

$$
\frac{d u^{+}}{d \xi}=\frac{1}{\kappa \xi}+r_{u} \frac{v_{u}^{+}}{\kappa \xi(1-\xi)} \frac{u_{* u}}{u_{*}}\left(1-\frac{u_{d}^{+}}{u_{u}^{+}}\right) u_{u}^{+} .
$$

Here, we denote

$$
A=r_{u} v_{u}^{+} \frac{u_{* u}}{u_{*}}\left(u_{u}^{+}-u_{d}^{+}\right)
$$

Then

$$
d u^{+}=\frac{1}{\kappa \xi} d \xi+\frac{A}{\kappa \xi(1-\xi)} d \xi
$$

Integration of (31) with respect to $\xi$ yields

$$
u^{+}=\frac{1}{\kappa} \ln \xi+\frac{A}{\kappa} \ln \xi-\frac{A}{\kappa} \ln (1-\xi)+C_{1} .
$$

In nonslip boundary conditions $u=0$ and then $\xi=\xi_{0}=y_{0} / h$ and thus $C_{1}=-(1 / \kappa) \ln \left(y_{0} / h\right)$

Finally, we obtained the corrected log equation as

$$
u^{+}=\frac{1}{\kappa} \ln \frac{y}{y_{0}}+\frac{A}{\kappa} \ln \left(\frac{y / h}{1-y / h}\right) .
$$

The value of $A$ used in (30) corrects the deviation of velocity from the log-law by considering upward and downward motion adequately. Thus, we can find the existence of the wake function from the newly derived log-law. Upward and downward motion can decompose turbulent flow into two distinct events, and it also indicates that if $u_{u}^{+}=u_{d}^{+}$, then the classic log-law can be obtained. Many researchers determine the different $\kappa$ and $B$ parameters in $u^{+}=1 / \kappa \ln u_{*} y / \nu+B$ from their experiments. Equation (33) implies that the main reason for such different results is not from different experimental conditions, but it is rather from upflow and downflow effects. It is necessary to verify (33) using experimental data.

\section{Experimental Set-Up}

The experiments were undertaken in a large-scale flow loop at the University of Wollongong, Australia, in which water was supplied from a head tank. The main components of this flume were the head tanks tail tank, glass water channel, recirculation pipe system, and two pumps as shown in Figure 1.

The head tank and the tail tank were constructed using stainless steel. The head tank was aligned centrally to the
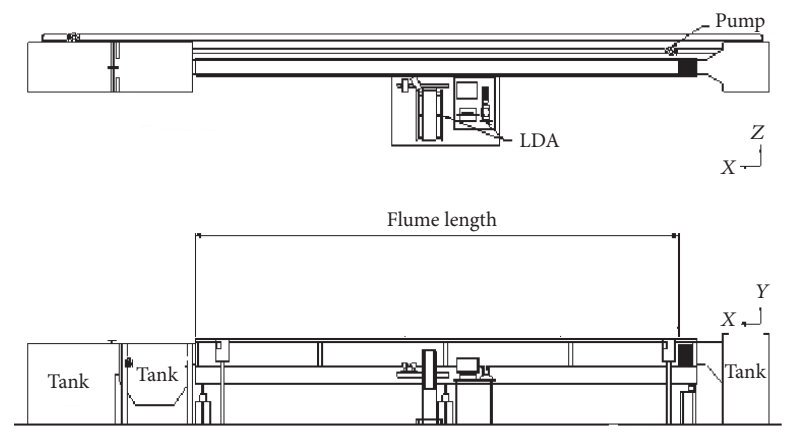

Figure 1: General view of the flume.

flume and symmetrical about the centre line of the channel so that the flow at the entrance of the channel was as uniform as possible. A honeycomb section was also located at the entrance to the flume. Through the addition of this honeycomb, the velocity distribution within the channel cross-section became increasingly uniform. The water depth through the flume was controlled by an adjustable tailgate in the downstream tail tank. The relative height of this tailgate was adjusted by rotating the attached handle. This was installed at the entrance of the tail tank. From the data recorded at these locations, it was possible to visually analyse the development of uniform flow in the channel prior to experiments.

The experimental flume was $10.5 \mathrm{~m}$ long, $0.3 \mathrm{~m}$ wide, and $0.45 \mathrm{~m}$ high with a slope set to a certain value for different experimental conditions. Experimental conditions are given in Table 1. A series of velocity profiles were measured in central line with different $b / h$ values. The flow depth $h$ was changed in order to examine the effect of aspect ratio in this study. Hence, a comprehensive set of high quality data, such as velocities, turbulence intensities, Reynolds stresses, and boundary shear stresses of the 3D turbulence structure, were also obtained.

Throughout the experimental process in this study the point velocities were measured using a Dantec twocomponent LDA system. It is a two-component configuration with a $60 \mathrm{~mm}$ optical fibre probe and a front lens with a $400 \mathrm{~mm}$ focal length. The system consists of a $300 \mathrm{~mW}$ continuous wave Argon-Ion laser and transmitting optics including a beam splitter Bragg-cell and signal processors. Green $(514.5 \mathrm{~nm})$ and blue $(488 \mathrm{~nm})$ components were used to measure the horizontal and vertical components of the 
velocity, respectively. The optics for $2 \mathrm{D} \mathrm{LDA}$ was arranged in off-axis backscatter mode. The backscatter mode of receiving a signal from a particle refers to the reflected light being captured by the receiving optics on the same side as the incident laser beams, with reflected light going back into the prove head through the front lens. The dimension of the measurement volume was approximately $0.189 \times 0.189 \times$ $3.97 \mathrm{~mm}$ for both colours when measured in air.

Hence, no calibration for LDA was necessary prior to the experiments. It is important to note that, during the measurement of velocity $u$ and $v$ by LDA, data acquisition of the components needed to be coincidental in time for all the data pairs $u(t)$ and $v(t)$. The signal processor is a Dantec Burst Spectrum Analyser (BSA) connected to an oscilloscope and a PC. The BSA converts the electrical signals, which are processed by the oscilloscope, into velocity data, which again are monitored online by a PC. Raw data exported from BSA can be processed into statistical values like mean velocities, root mean squared values of velocity fluctuations, and Reynolds stresses.

\section{Results and Discussion}

The velocity profiles for a smooth rectangular channel are plotted in Figures 2-7, and the velocity profiles predicted by DML-Law, MLW-Law, and CML-Law are used for comparison to experimental data. These figures show that the log-law is able to predict all experimental data in the inner region. It can be clearly found that the wake function indeed exists in uniform flow. However, DML-Law, MLW-Law, and CMLLaw are not able to agree with the experimental data properly in the outer region; that is, $y / h>0.6$. It is observed that the measured values for all runs are relatively higher than the calculated results from DML-Law in the outer region. It is impossible to improve the velocity profiles predicted by the DML-Law by adjusting parameter $\alpha$, so further improvement of (5) is recommended. The DML-Law seems to be able to predict the velocity-dip phenomenon, but with inaccurate maximum velocities. Accurate predictions of the velocity-dip phenomenon therefore require larger values of $\alpha$.

It can be seen from Figures 2-4 that the MLW-Law and CML-Law significantly overestimate the velocity profile in the outer region. Both of these two methods seem not to be a suitable predictor for channels with $b / h>3$ in the outer region. In particular, the CML-Law is important for an inadequate prediction of the dip phenomenon with $b / h>3$, and $\Pi=0.55$ seems to be too small to improve predictions. Figures 5-7 show that the MLW-Law and CMLLaw are more accurate in predicting the velocity profiles for $b / h<3$. The effect of each term in the MLW-Law and CMLLaw is not significant. In general, the average differences between the MLW-Law, CML-Law, and DML-Law theories and the current measurements give relatively large averaged $E$ values of $7.1 \%, 5.9 \%$, and $5.7 \%$, respectively. The average differences between measurements and the current approach give a relatively small average $E$ value of $1.0 \%$.

Figures 2-7 present comparisons of predicted velocity profiles obtained by (33) with experimental data, and we note that the parameter $A$ in (33) is an important value

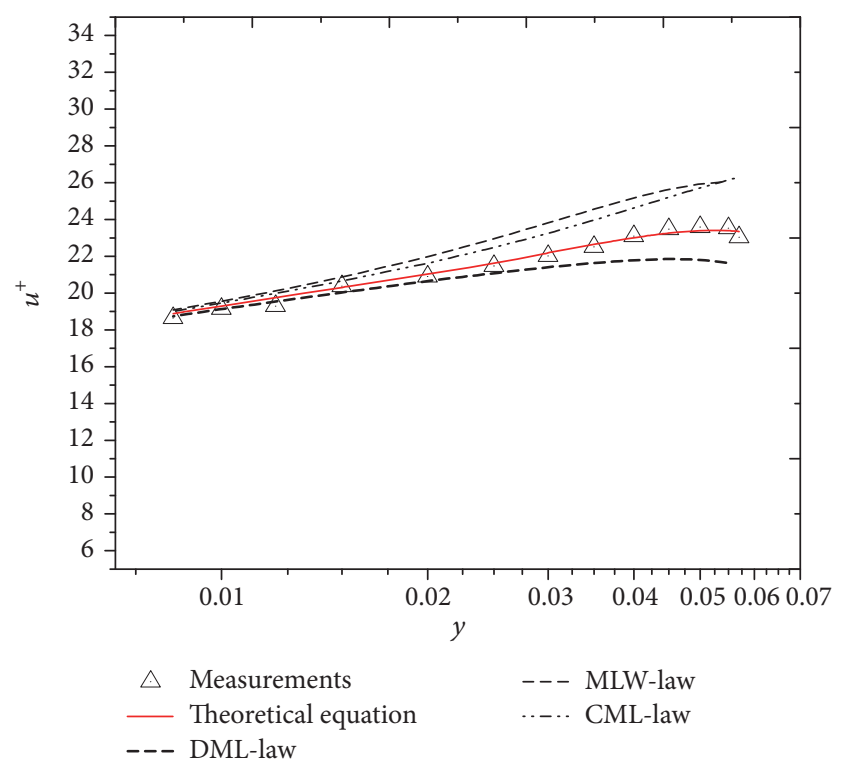

FIGURE 2: Comparison of velocity profiles obtained from theoretical equation (see (33)), DML, MLW, and CML with experimental data (Run 1).

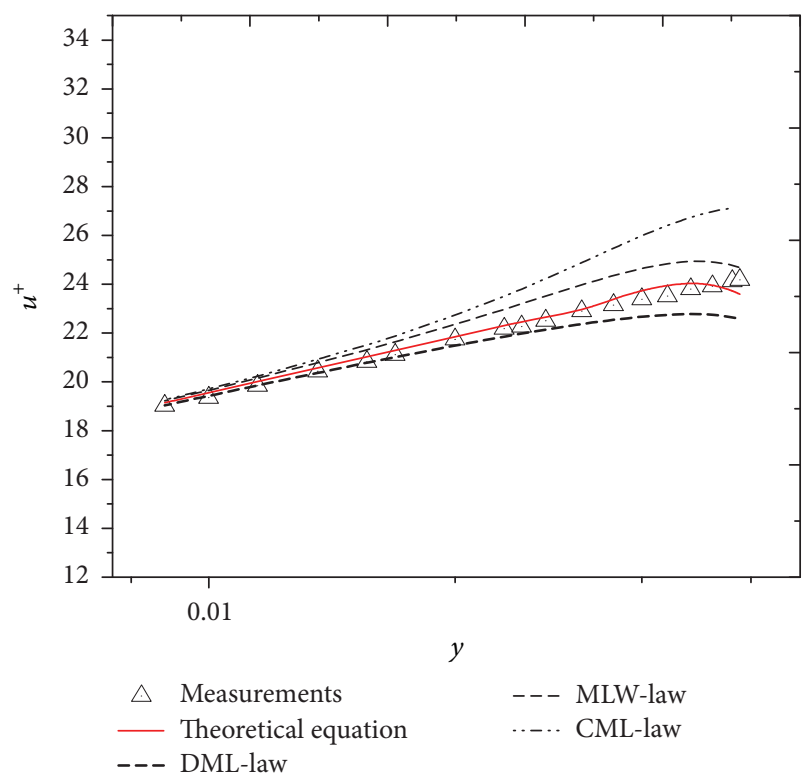

FIGURE 3: Comparison of velocity profiles obtained from theoretical equation (see (33)), DML, MLW, and CML with experimental data (Run 2).

in improving the predicted velocity profiles. Thus, to plot the velocity distribution in the entire flow region using the proposed theoretical equation, it was necessary to determine the value of $A$ in (30) accurately. The values of $r_{u}$ and $v_{u}^{+}$in (30) can be easily calculated using (17) and (21), respectively, from a given data set of upflow velocities. We also note that $u_{*}$ in (30) is the mean shear stress; that is, $u_{*}=\sqrt{g R S}$. Furthermore, in a plot of measured upflow/downflow velocity against $\log y$ for a profile along the central line, the unknowns $u_{* u}$ and $u_{* d}$ can be evaluated from its slope, respectively. 


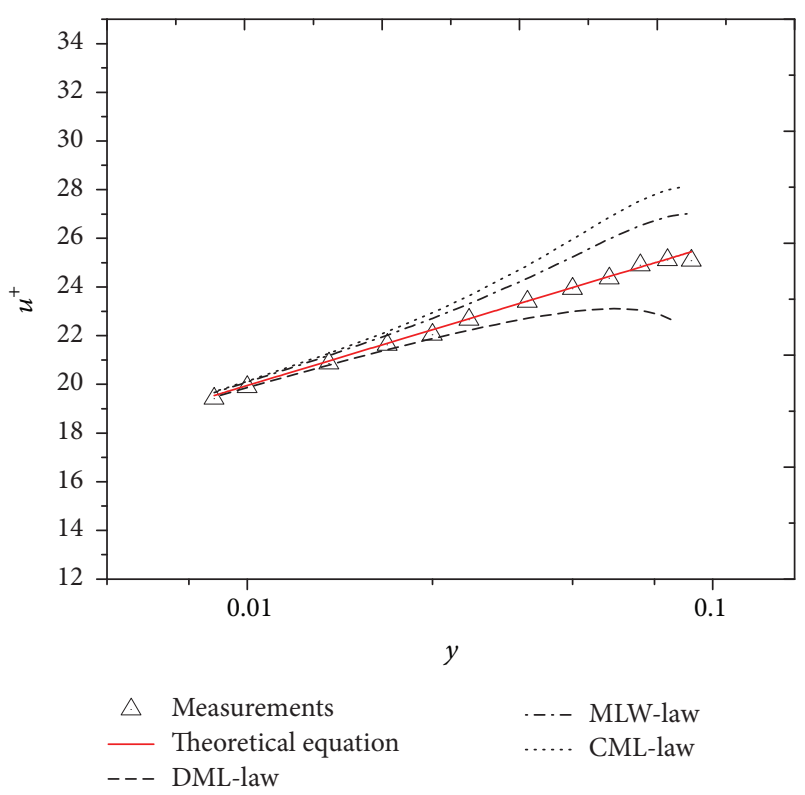

FIgURE 4: Comparison of velocity profiles obtained from theoretical equation (Eq. (33)), DML, MLW, and CML with experimental data (Run 3).

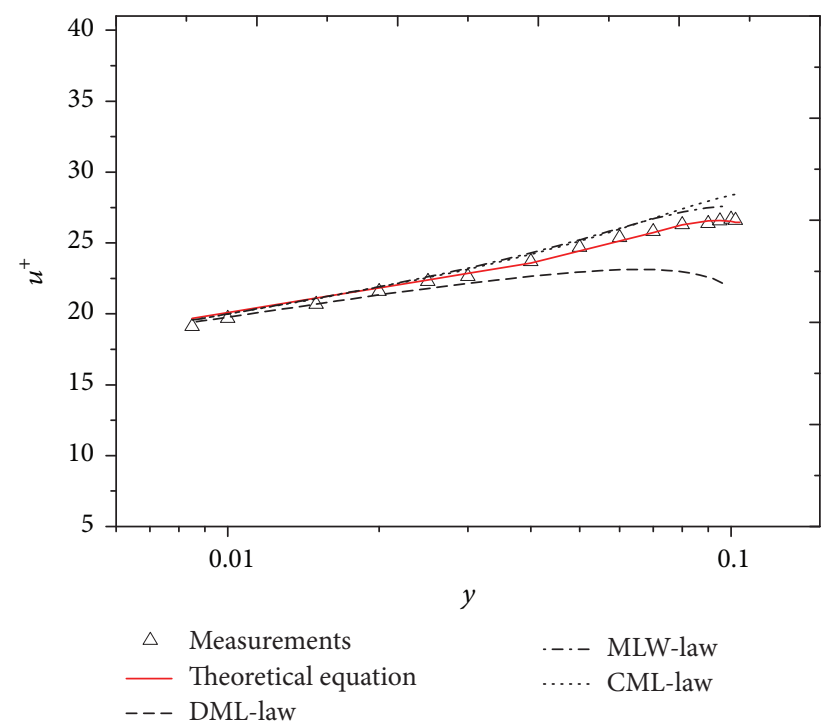

Figure 5: Comparison of velocity profiles obtained from theoretical equation (see (33)), DML, MLW, and CML with experimental data (Run 4).

The values of $r_{u}, v_{u}^{+}, u_{*}, u_{* u}$, and $u_{* d}$ used in (30) are then calculated and included in Tables 2 and 3.

The experimental data of $u_{u}$ and $u_{d}$ scale with $u_{* u}$ and $u_{* d}$, and then $u_{u}^{+}$and $u_{d}^{+}$can be calculated, respectively. In (30), the ratio of $u_{d}^{+}$and $u_{u}^{+}$is larger than 1 in the outer region, as shown in Figure 8; thus, the values of $A$ are all negative in the outer region. Hence, the wake function $(A / \kappa) \ln (y / h /(1-$ $y / h)$ ), in (33) would also be a negative value in the outer region, implying that the velocity from (33) must be lower than that predicted by the log-law, or there is a velocity

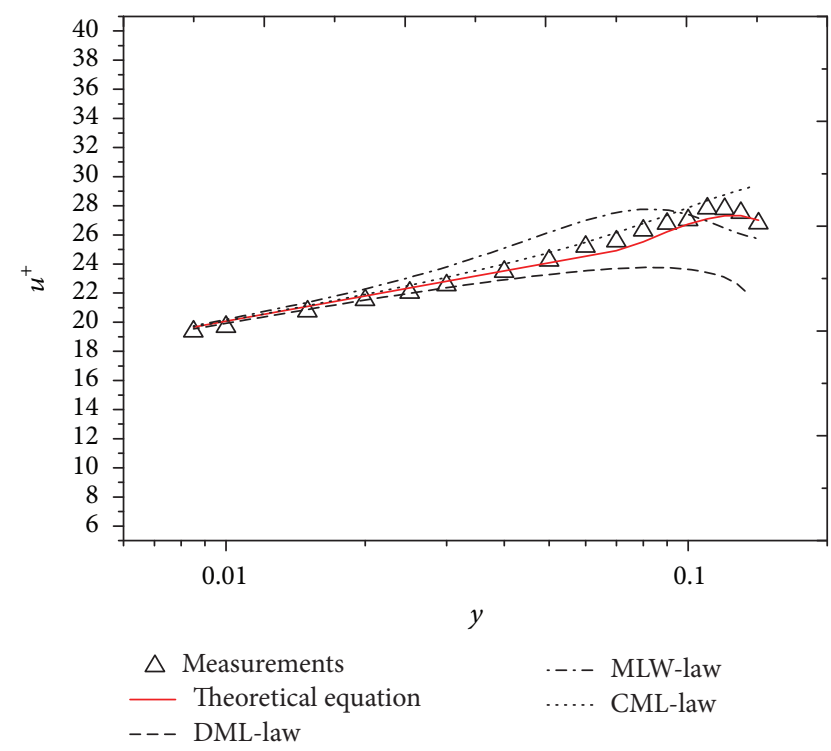

FIGURE 6: Comparison of velocity profiles obtained from theoretical equation (see (33)), DML, MLW, and CML with experimental data (Run 5).

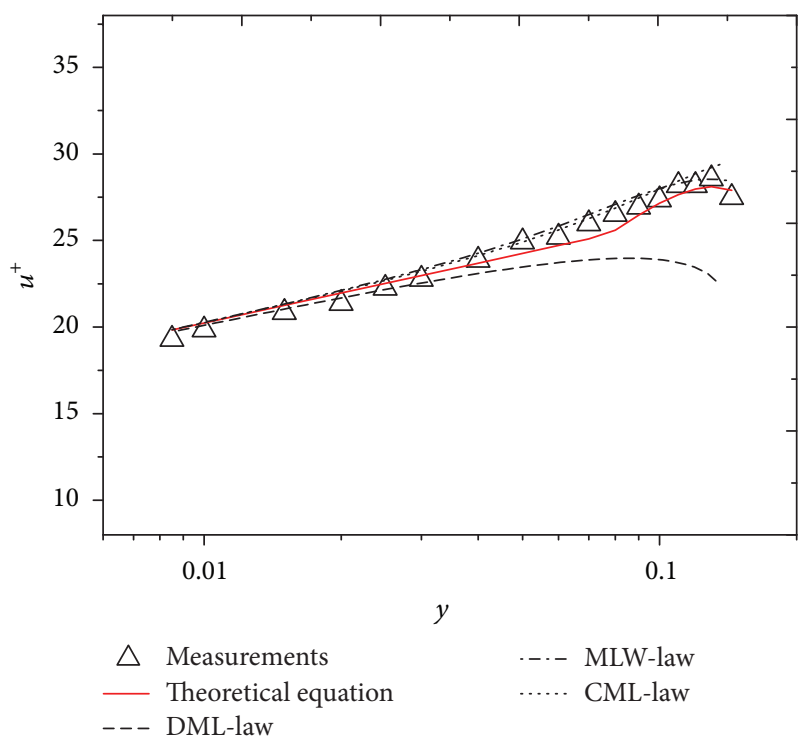

FIGURE 7: Comparison of velocity profiles obtained from theoretical equation (see (33)), DML, MLW, and CML with experimental data (Run 6).

dip near the water surface. Then, it is clearly indicated that downward motions will mainly cause the wake strength. In other words, in 2D flows there indeed exists a wake function, which is caused by variations in the wall-normal velocity. These different $A$ values have been applied in the proposed theoretical equation and have amounted to approximately $1.5 \%$ errors from the velocity encountered in the experiments. They are much smaller than the corresponding errors for other comparisons. It will be seen that the value of $A$ used in (30) adequately accounts for the errors in comparison. 
TABLE 2: Summary of the main parameters in (30) (Run 1, Run 3, and Run 4).

\begin{tabular}{|c|c|c|c|c|c|c|c|c|}
\hline \multicolumn{3}{|c|}{$h=0.065 \mathrm{~m}$} & \multicolumn{3}{|c|}{$h=0.100 \mathrm{~m}$} & \multicolumn{3}{|c|}{$h=0.110 \mathrm{~m}$} \\
\hline$u_{* u}$ & $u_{* d}$ & $u_{*}$ & $u_{* u}$ & $u_{* d}$ & $u_{*}$ & $u_{* u}$ & $u_{* d}$ & $u_{*}$ \\
\hline 0.021 & 0.020 & 0.022 & 0.027 & 0.026 & 0.026 & 0.029 & 0.029 & 0.025 \\
\hline$y / h$ & $r_{u}$ & $v_{u}^{+}$ & $y / h$ & $r_{u}$ & $v_{u}^{+}$ & $y / h$ & $r_{u}$ & $v_{u}^{+}$ \\
\hline 0.131 & 0.460 & 1.182 & 0.085 & 0.504 & 1.375 & 0.077 & 0.496 & 1.274 \\
\hline 0.154 & 0.477 & 0.911 & 0.100 & 0.503 & 0.790 & 0.091 & 0.477 & 0.863 \\
\hline 0.185 & 0.492 & 0.935 & 0.150 & 0.482 & 0.768 & 0.136 & 0.507 & 0.909 \\
\hline 0.231 & 0.520 & 0.855 & 0.200 & 0.505 & 0.824 & 0.182 & 0.505 & 0.898 \\
\hline 0.308 & 0.458 & 1.089 & 0.250 & 0.470 & 0.750 & 0.227 & 0.469 & 0.870 \\
\hline 0.385 & 0.457 & 0.617 & 0.300 & 0.488 & 0.680 & 0.273 & 0.494 & 0.796 \\
\hline 0.462 & 0.496 & 0.621 & 0.400 & 0.478 & 0.423 & 0.364 & 0.458 & 0.761 \\
\hline 0.538 & 0.446 & 0.636 & 0.500 & 0.513 & 0.559 & 0.455 & 0.458 & 0.695 \\
\hline 0.615 & 0.443 & 0.547 & 0.600 & 0.489 & 0.382 & 0.545 & 0.493 & 0.558 \\
\hline 0.692 & 0.489 & 0.523 & 0.700 & 0.487 & 0.423 & 0.636 & 0.476 & 0.533 \\
\hline 0.769 & 0.514 & 0.696 & 0.800 & 0.489 & 0.393 & 0.727 & 0.474 & 0.368 \\
\hline 0.846 & 0.471 & 0.832 & 0.900 & 0.504 & 0.364 & 0.818 & 0.512 & 0.312 \\
\hline \multirow[t]{3}{*}{0.877} & 0.471 & 0.752 & & & & 0.864 & 0.473 & 0.302 \\
\hline & & & & & & 0.909 & 0.481 & 0.295 \\
\hline & & & & & & 0.927 & 0.496 & 0.253 \\
\hline
\end{tabular}

TABLE 3: Summary of the main parameters in (30) (Run 2, Run 5, and Run 6).

\begin{tabular}{|c|c|c|c|c|c|c|c|c|}
\hline \multicolumn{3}{|c|}{$h=0.085 \mathrm{~m}$} & \multicolumn{3}{|c|}{$h=0.15 \mathrm{~m}$} & \multicolumn{3}{|c|}{$h=0.16 \mathrm{~m}$} \\
\hline$u_{* u}$ & $u_{* d}$ & $u_{*}$ & $u_{* u}$ & $u_{* d}$ & $u_{*}$ & $u_{* u}$ & $u_{* d}$ & $u_{*}$ \\
\hline 0.026 & 0.024 & 0.024 & 0.026 & 0.025 & 0.025 & 0.028 & 0.028 & 0.027 \\
\hline$y / h$ & $r_{u}$ & $v_{u}^{+}$ & $y / h$ & $r_{u}$ & $v_{u}^{+}$ & $y / h$ & $r_{u}$ & $v_{u}^{+}$ \\
\hline 0.100 & 0.491 & 0.915 & 0.057 & 0.508 & 1.015 & 0.055 & 0.485 & 0.989 \\
\hline 0.118 & 0.475 & 0.877 & 0.067 & 0.500 & 1.214 & 0.065 & 0.482 & 1.018 \\
\hline 0.141 & 0.470 & 0.900 & 0.100 & 0.468 & 1.248 & 0.097 & 0.522 & 1.179 \\
\hline 0.176 & 0.483 & 0.792 & 0.133 & 0.497 & 1.271 & 0.129 & 0.451 & 1.268 \\
\hline 0.212 & 0.496 & 0.862 & 0.167 & 0.514 & 1.248 & 0.161 & 0.545 & 1.229 \\
\hline 0.235 & 0.478 & 0.862 & 0.200 & 0.535 & 1.286 & 0.194 & 0.503 & 1.207 \\
\hline 0.294 & 0.500 & 0.750 & 0.267 & 0.478 & 1.347 & 0.258 & 0.482 & 1.218 \\
\hline 0.353 & 0.467 & 0.685 & 0.333 & 0.513 & 1.267 & 0.323 & 0.502 & 1.218 \\
\hline 0.376 & 0.478 & 0.735 & 0.400 & 0.521 & 1.401 & 0.387 & 0.485 & 1.186 \\
\hline 0.412 & 0.479 & 0.712 & 0.467 & 0.513 & 1.450 & 0.452 & 0.484 & 1.171 \\
\hline 0.471 & 0.473 & 0.538 & 0.533 & 0.476 & 1.355 & 0.516 & 0.473 & 1.046 \\
\hline 0.529 & 0.510 & 0.427 & 0.600 & 0.449 & 1.294 & 0.581 & 0.443 & 0.904 \\
\hline 0.588 & 0.483 & 0.527 & 0.667 & 0.456 & 1.172 & 0.645 & 0.481 & 0.832 \\
\hline 0.647 & 0.463 & 0.488 & 0.733 & 0.496 & 1.137 & 0.710 & 0.489 & 0.839 \\
\hline 0.706 & 0.468 & 0.415 & 0.800 & 0.449 & 1.218 & 0.774 & 0.501 & 0.721 \\
\hline 0.765 & 0.450 & 0.454 & 0.867 & 0.519 & 1.065 & 0.839 & 0.514 & 0.446 \\
\hline 0.824 & 0.478 & 0.288 & 0.947 & 0.609 & 1.477 & 0.929 & 0.500 & 0.957 \\
\hline 0.847 & 0.496 & 0.250 & & & & & & \\
\hline
\end{tabular}

Similarly, $\beta=\left(u_{u}^{+}-u_{d}^{+}\right)$can be determined from experimental data. The above analysis applies to (30) in which all parameters are found, and thus the relationship between $A$ and $\beta$ can then be plotted in Figure 9. The dimensionless profiles of $\beta$ collapse reasonably well between the experiments, indicating that the relationship between $A$ and $\beta$ for different water depths are similar. Therefore, an empirical equation can be fitted using data $A$ and $\beta$, as shown in Figure 9.

The parameter $A$ in (33) is an important value for improving the predicted velocity profiles. To simplify the calculation of $A$ in practice, Figure 8 depicts the fitting of a regression equation. For a particular channel (constant aspect ratio), it is easier to check the value of $u_{d}^{+} / u_{u}^{+}$from Figure 8 to 


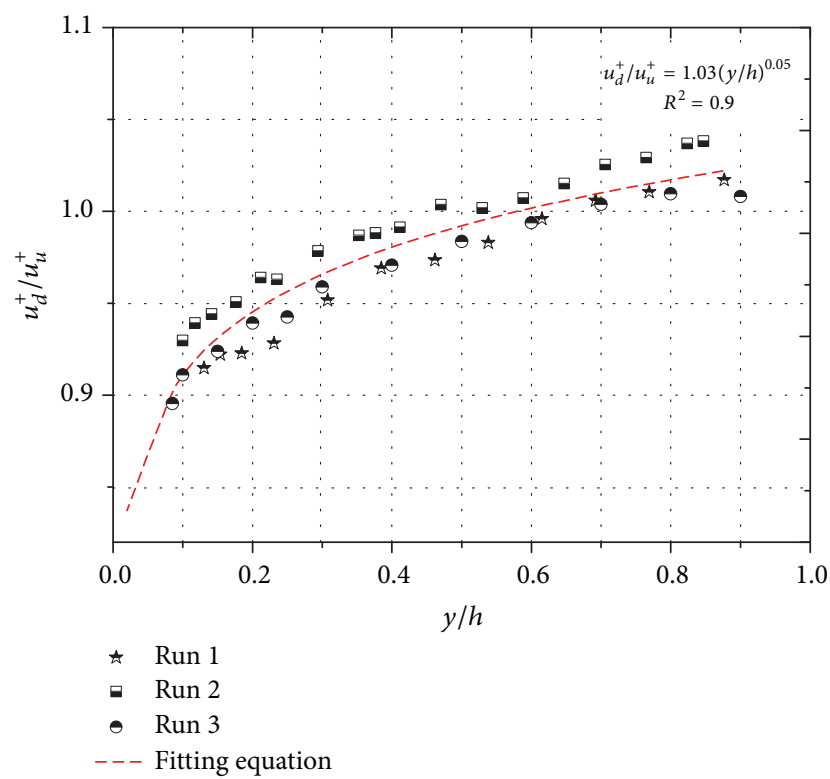

FIGURE 8: Variation of $u_{d}^{+} / u_{u}^{+}$with $y / h$.

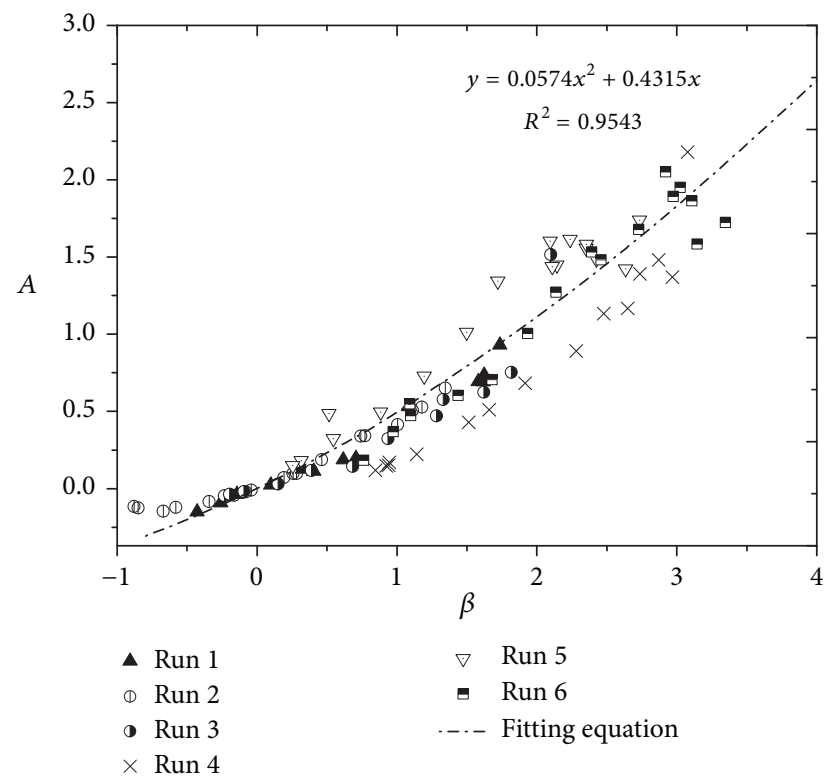

FIGURE 9: Variation of $\beta$ with parameter $A$.

obtain the value of $\beta$; thus facilitating obtaining the value of $A$ in Figure 9.

\section{Conclusions}

In this study, we theoretically investigated conditionally averaged turbulent structures for upward and downward motions in a $2 \mathrm{D}$ uniform flow. The connection between wake law and up/down events was also explored. The following conclusions can be drawn from the foregoing analysis:

(1) In 2D flows, the wake function is caused by the additional momentum flux. By considering up/down events, it explores the underlying mechanism of Coles' wake law and establishes a relationship between the wake function and up/down events.

(2) Applying the proposed theoretical equation to experimental measurements, good agreement is achieved between the theoretical equation and the measurements. The theoretical results show that the momentum flux caused by the conditional up/down events leads to deviation of velocity from the log-law.

(3) The DML-Law, MLW-Law, and CML-Law models recorded in the literature were examined in this study. Figures 2-7 show that all of these models are able to predict all experimental data in the inner region. However, DML-Law, MLW-Law, and CML-Law are not able to agree with the experimental data properly in the outer region. It is clearly found that the wake function indeed exists in uniform flow.

\section{Conflicts of Interest}

The authors declare that they have no conflicts of interest.

\section{Acknowledgments}

The authors express gratitude for the financial support from the National Key Research and Development Plan (Grant no. 2016YFC0400207), the National Natural Science Foundation of China (Grant no. 51509248), the Chinese Universities Scientific Fund (Grant nos. 2015QC025, 2017SL001), and the Scientific Research and Experiment of Regulation Engineering for the Songhua River Mainstream in Heilongjiang Province, P. R. of China (Grant no. SGZL/KY-12).

\section{References}

[1] H. Afzalimehr and F. Anctil, "Velocity distribution and shear velocity behaviour of decelerating flows over a gravel bed," Canadian Journal of Civil Engineering, vol. 26, no. 4, pp. 468475, 1999.

[2] Y. Han, S.-Q. Yang, N. Dharmasiri, and M. Sivakumar, "Effects of sample size and concentration of seeding in LDA measurements on the velocity bias in open channel flow," Flow Measurement and Instrumentation, vol. 38, pp. 92-97, 2014.

[3] T. Stoesser, R. McSherry, and B. Fraga, "Secondary currents and turbulence over a non-uniformly roughened open-channel bed," Water (Switzerland), vol. 7, no. 9, pp. 4896-4913, 2015.

[4] Y. Han, S.-Q. Yang, N. Dharmasiri, and M. Sivakumar, "Experimental study of smooth channel flow division based on velocity distribution," Journal of Hydraulic Engineering, vol. 141, no. 4, Article ID 06014025, 2015.

[5] S.-Q. Yang, S.-K. Tan, S.-Y. Lim, and S.-F. Zhang, "Velocity distribution in combined wave-current flows," Advances in Water Resources, vol. 29, no. 8, pp. 1196-1208, 2006.

[6] R. Absi, "An ordinary differential equation for velocity distribution and dip-phenomenon in open channel flows," Journal of Hydraulic Research, vol. 49, no. 1, pp. 82-89, 2011.

[7] A. J. Smits, B. J. McKeon, and I. Marusic, "High-Reynolds number wall turbulence," Annual Review of Fluid Mechanics, vol. 43, pp. 353-375, 2011. 
[8] I. Nezu, "Open-channel flow turbulence and its research prospect in the 21st century," Journal of Hydraulic Engineering, vol. 131, no. 4, pp. 229-246, 2005.

[9] D. Coles, "The law of the wake in the turbulent boundary layer," Journal of Fluid Mechanics, vol. 1, pp. 191-226, 1956.

[10] A. H. Cardoso, W. H. Graf, and G. Gust, "Uniform flow in a smooth open channel," Journal of Hydraulic Research, vol. 27, no. 5, pp. 603-616, 1989.

[11] B. A. Kironoto, W. H. Graf, and Reynolds, "Turbulence characteristics in rough non-uniform open-channel flow," Proceedings of the Institution of Civil Engineers - Water and Maritime Engineering, vol. 112, no. 4, pp. 336-348, 1995.

[12] J. Guo and P. Y. Julien, "Modified log-wake law for turbulent flow in smooth pipes," Journal of Hydraulic Research, vol. 41, no. 5, pp. 493-501, 2003.

[13] S.-Q. Yang, S.-Y. Lim, and J. A. McCorquodale, "Investigation of near wall velocity in 3-D smooth channel flows," Journal of Hydraulic Research, vol. 43, no. 2, pp. 149-157, 2005.

[14] N. L. Coleman, "Effects of Suspended Sediment on the Open-Channel Velocity Distribution," Water Resources Research, vol. 22, no. 10, pp. 1377-1384, 1986.

[15] I. Nezu and W. Rodi, "Open-channel flow measurements with a laser doppler anemometer," Journal of Hydraulic Engineering, vol. 112, no. 5, pp. 335-355, 1986.

[16] M. S. Kirkgöz, "Turbulent velocity profiles for smooth and rough open channel flow," Journal of Hydraulic Engineering, vol. 115, no. 11, pp. 1543-1561, 1989.

[17] S.-Q. Yang and J. A. McCorquodale, "Determination of boundary shear stress and Reynolds shear stress in smooth rectangular channel flows," Journal of Hydraulic Engineering, vol. 130, no. 5, pp. 458-462, 2004.

[18] S.-Q. Yang, S.-K. Tan, and S.-Y. Lim, "Velocity distribution and dip-phenomenon in smooth uniform open channel flows," Journal of Hydraulic Engineering, vol. 130, no. 12, pp. 1179-1186, 2004.

[19] I. Nezu and H. E. D. Nakagawa, Turbulence in Open-Channel Flows, Balkema, Rotterdam, The Netherlands, 1993.

[20] H. Bonakdari, F. Larrarte, L. Lassabatere, and C. Joannis, "Turbulent velocity profile in fully-developed open channel flows," Environmental Fluid Mechanics, vol. 8, no. 1, pp. 1-17, 2008.

[21] R. Absi, "Comments on "turbulent velocity profile in fullydeveloped open channel flows", Environmental Fluid Mechanics, vol. 8, no. 4, pp. 389-394, 2008.

[22] J. H. Pu, H. Bonakdari, L. Lassabatère, C. Joannis, and F. Larrarte, "Profil de vitesses turbulent : une nouvelle loi pour les canaux étroits," La Houille Blanche, no. 3, pp. 65-70, 2010.

[23] H. Afzalimehr, R. Moghbel, J. Gallichand, and J. Sui, "Investigation of turbulence characteristics in channel with dense vegetation," International Journal of Sediment Research, vol. 26, no. 3, pp. 269-282, 2011.

[24] M. Cellino and U. Lemmin, "Influence of coherent flow structures on the dynamics of suspended sediment transport in open-channel flow," Journal of Hydraulic Engineering, vol. 130, no. 11, pp. 1077-1088, 2004.

[25] S.-Q. Yang, "Conditionally averaged turbulent structures in 2D channel flow," Proceedings of the Institution of Civil Engineers: Water Management, vol. 163, no. 2, pp. 79-88, 2010. 


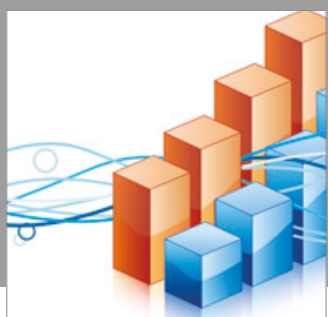

Advances in

Operations Research

vatersals

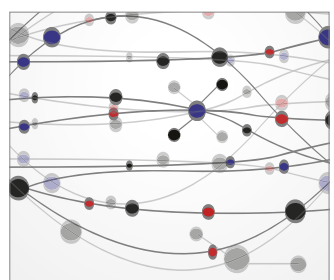

\section{The Scientific} World Journal
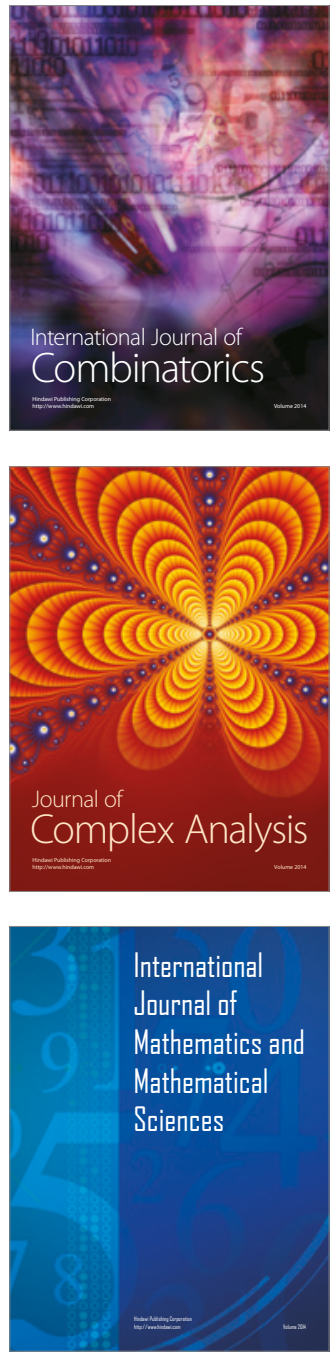
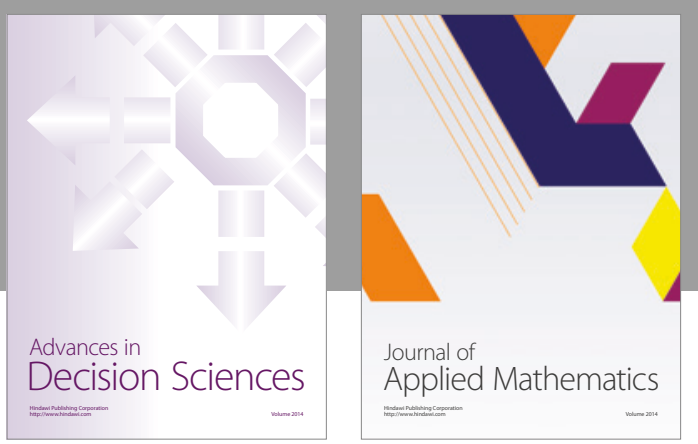

Algebra

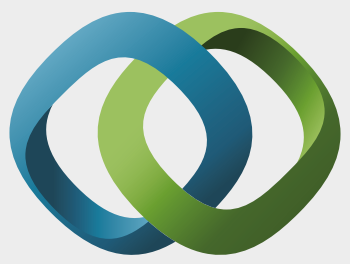

\section{Hindawi}

Submit your manuscripts at

https://www.hindawi.com
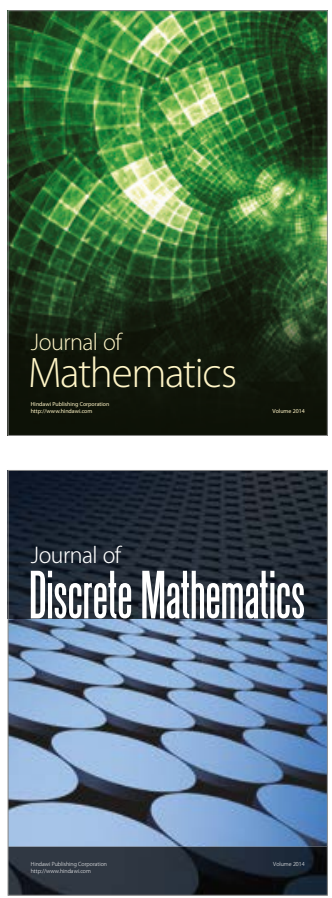

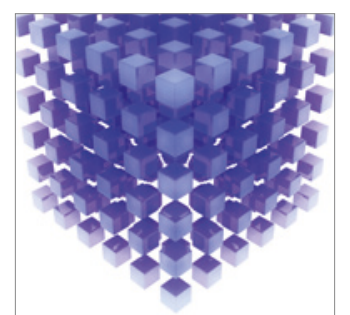

Mathematical Problems in Engineering
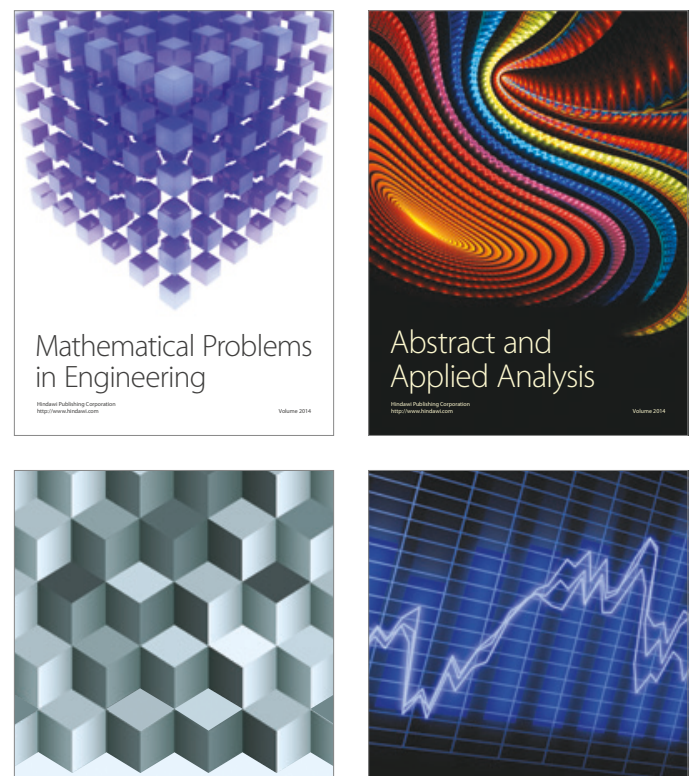

Journal of

Function Spaces

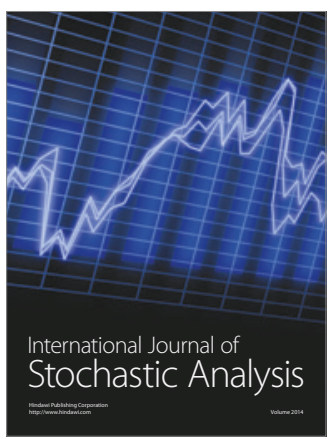

Probability and Statistics
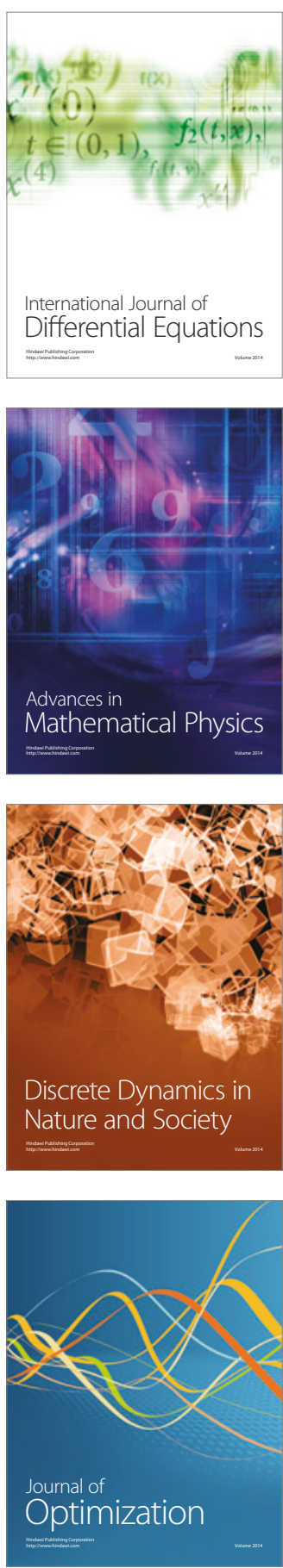